\title{
Rechtsstaatlichkeit, Good Governance und Entwicklung
}

\author{
Von Wolfgang Muno, Erfurt*
}

\section{Einleitung}

Rechtsstaatlichkeit bzw. „Rule of law“ (als Synonyme verstanden und hier auch so benutzt) ${ }^{1}$ ist ein Modethema der Außen- und Entwicklungspolitik geworden. Fast überall geht es um Rechtsstaatlichkeit, externe Demokratie- und Rechtsstaatsförderung oder Rule of Law als Bestandteil von Good Governance. Dabei wird Rechtsstaatlichkeit oft als Allheilmittel für viele Weltprobleme angesehen. Ob die Beilegung von Bürgerkriegen, Demokratisierungsprobleme, Entwicklung oder Armutsbekämpfung - Rechtsstaatlichkeit wird als ein, wenn nicht der entscheidende Faktor aufgeführt. „The concept is suddenly everywhere“, schreibt Thomas Carothers in seinem Artikel „The Rule-of-Law Revival”. ${ }^{2}$ Die G8-Außenminister verabschiedeten 2007 eine „Erklärung zur Förderung der Rechtsstaatlichkeit“; 3 in der OECD, der EU, bei USAID, auch im BMZ, in der GIZ und im Auswärtigen Amt sind Rechtsstaatlichkeit und Rechtsstaatsförderung „zu einem zentralen Topos des entwicklungspolitischen und entwicklungsökonomischen Diskurses ebenso wie der Außen- und Sicherheitspolitik geworden", wie Riegner und Wischmeyer schreiben. ${ }^{4}$

Die Diskussion über Rechtsstaatlichkeit und Entwicklung ist dabei nicht neu. Von Modernisierungstheorien inspiriert, beherrschte in den 50er, 60er und 70er Jahren des 20. Jahrhundert das „Law and Development Movement“ die Debatte, in den 90er Jahren „morphte“ sie zu „Good Governance“. 5 Eine besondere Rolle in Entwicklungstheorie wie Entwick-

* Wolfgang Muno, Dr. phil., Vertretungsprofessor für Vergleichende Regierungslehre an der Universität Erfurt, Willy Brandt School of Public Policy. E-Mail: wolfgang.muno@uni-erfurt.de. Der vorliegende Beitrag ist im Rahmen des DFG-Projektes „Rechtsstaat und informelle Institutionen - Osteuropa und Lateinamerika im Vergleich" an der Universität Würzburg entstanden. Ich danke meinen Kollegen in Würzburg für zahlreiche und sogar meist konstruktive Hinweise.

1 Vgl. dazu auch Helmuth Schulze-Fielitz, Zur Geltung des Rechtsstaates: Zwischen Kulturangemessenheit und universellem Anspruch, Zeitschrift für Vergleichende Politikwissenschaft 5, 1 (2011), S. 1-23.

2 Thomas Carothers, The Rule-of-Law Revival, Foreign Affairs 77, 2 (1998), S. 95-106.

3 Erklärung der Außenminister der G8-Staaten vom 30. Mai 2007, http://v 1.bitv-test.de/dateien/ pdf_test/834/AM-Erkl-Rechtstaatlichkeit.pdf.).

4 Michael Riegner/Thomas Wischmeyer, "Rechtliche Zusammenarbeit" mit Transformations- und Entwicklungsländern als Gegenstand öffentlich-rechtlicher Forschung, Der Staat 50, 3 (2011), S. 436-467, 436.

5 Brian Tamanaha, The Primacy of Society and the Failures of Law and Development: Decades of Stubborn Refusal to Learn, Faculty Research paper Series 10-03-02 (2010), S. 13. Zu "Law and Development" siehe auch Brun-Otto Bryde, Constitutional Law in "old" and "new" Law and Development, Verfassung und Recht in Übersee (VRUE) 41, 1 (2008), S. 10-16; Franz von Benda-Beckmann, Law and Development im Wandel, VRUE 41, 1 (2008), S. 295- 308, Michael Riegner/Philipp 
lungspolitik kam dabei der Weltbank zu, die das „Good Governance“ Konzept und damit auch explizit Rule of Law, anknüpfend an institutionenökonomische Überlegungen, propagierte und auch aktiv in der Rechtsstaatsförderung tätig ist. Laut eigenen Angaben wurden seit Anfang der 1990er Jahre über 30 einschlägige Projekte mit einem gesamten Finanzvolumen von über 850 Millionen US-Dollar gefördert, Trubek spricht von 330 Projekten und 2,9 Milliarden US-Dollar, Riegner und Wischmeyer von 3,8 Milliarden, bei einem sehr weiten Rechtsstaatsverständnis sogar von 12 Milliarden US-Dollar. ${ }^{6}$

Im Folgenden soll die Diskussion über Rechtsstaatlichkeit, Good Governance und Entwicklung nachgezeichnet werden. Insbesondere die Rolle der Weltbank steht dabei im Mittelpunkt. Mit den World Governance Indicators (im Folgenden WGI) hat die Weltbank in den 1990er Jahren ein sehr einflussreiches Unternehmen gestartet, Governance und damit auch Rule of Law zu messen. Empirischen Studien in der Entwicklungsökonomik und der Politikwissenschaft, aber auch Entwicklungsorganisationen stützen sich auf die WGI, um die Bedeutung von Rechtsstaatlichkeit für Entwicklung quantitativ zu bestimmen. Deshalb ist es von besonderem Interesse, die WGI und insbesondere die Messung von Rule of Law im Rahmen der WGI genauer zu untersuchen.

\section{Rule of Law und Governance}

Es existiert eine Vielzahl verschiedener Definitionen von Rechtsstaatlichkeit; allein für die deutsche verfassungsrechtliche Diskussion erwähnt Schulze-Fielitz 141 Konkretisierungen und Auslegungen des Rechtsstaatsprinzips. ${ }^{7}$ In der internationalen Diskussion über Rule of Law werden ,thin“ und „thick“, dünnere und dichtere Definitionen unterschieden. Erstere beschränken sich auf formale Aspekte, letztere betonen materielle Aspekte, bei einigen ganz „dicken“ finden sich auch Demokratie- und Verteilungsaspekte. ${ }^{8}$ Eng verbunden mit Rule of Law ist „Governance”. Rechtsstaatlichkeit wird als Bestandteil von Governance, alternativ

Dann, "Recht und Entwicklung" als Gegenstand der Juristenausbildung: Konturen und Didaktik eines intra- und interdisziplinär vernetzten Studienfachs, VRUE 41, 1 (2008), S. 309-335, Jörg Menzel, Kambodscha und der Kampf ums Recht. Eine Fallstudie zu „Recht und Entwicklung“, VRUE 41, 1 (2008), S. 387-406, David M. Trubek, The "Rule of Law" in Development Assistance: Past, Present, and Future, in: David M. Trubek, Alvaro Santos (Hg.), The New Law and Economic Development. A Critical Appraisal, Cambridge 2006, S. 74-94 sowie Michael J. Trebilcock/Ronald J. Daniels, Rule of Law Reform and Development. Charting the Fragile Path of Progress, Cheltenham 2008.

6 Vgl. Trubek2006 (Fn. 5), Riegner/Wischmeyer 2011 (Fn. 4) sowie die Homepage der Weltbank (http:// web.worldbank.org/WBSITE/EXTERNAL/TOPICS/EXTLAWJUSTICE/0,menuPK: 445640 pagePK:149018 piPK:149093 theSitePK:445634,00.html).

7 Vgl. Schulz-Fielitz 2011 (Fn. 1), siehe auch Dieter Grimm, Stufen der Rechtsstaatlichkeit. Zur Exportfähigkeit einer westlichen Errungenschaft, JZ 2009, S. 596-600.

8 Brian Tamanaha, On the Rule of Law, Cambridge 2004; jüngst Jørgen Møller / Svend-Erik Skaaning, Systematizing Thin and Thick Conceptions of the Rule of Law, Paper prepared for presentation at the APSA Annual Meeting, Washington, D.C., September 2-5 (2010). 
als zentrale Governance-Ressource gesehen. ${ }^{9}$ Der Begriff „Governance“ ist eine der modischen Schöpfungen der Wissenschaft, eine „catchphrase“, wie Werner Jann schreibt, deren Verwendung in wissenschaftlichen Publikationen seit 1990 um das 30fache gestiegen sei. ${ }^{10}$ Schuppert schlägt eine Schneise in die Begriffswucherungen und identifiziert vier Wurzeln: ${ }^{11}$ politik- und verwaltungswissenschaftliche Diskussionen, den Transnationalismus der Teildisziplin Internationale Beziehungen (IB), die Institutionenökonomik und die Weltbank. Diese vier Wurzeln können ganz offensichtlich in zwei Diskussionsstränge zusammengefasst werden, einmal einen politikwissenschaftlichen, zum zweiten einen ökonomischen Strang.

Der politikwissenschaftliche Strang umfasst den IB-Ansatz des Transnationalismus, der

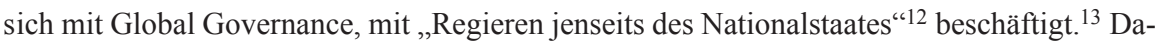
neben gibt es einen stärker theoretisch-komparativ orientierten Ansatz der Policy-Analyse. Zentrale Vertreter dieses Ansatzes in Deutschland sind Renate Mayntz und Fritz Scharpf. ${ }^{14}$ Governance wird von Mayntz weit und umfassend definiert als „das Gesamt aller nebeneinander bestehenden Formen der kollektiven Regelung gesellschaftlicher Sachverhalte: von der institutionalisierten zivilgesellschaftlichen Selbstregelung über verschiedene Formen des Zusammenwirkens staatlicher und privater Akteure bis hin zu hoheitlichem Handeln staatlicher Akteure". 15

Der ökonomische Strang kommt aus der Institutionenökonomik. Ronald Coase ${ }^{16}$ und Oliver Williamson ${ }^{17}$ haben mit dem Begriff Governance auf Organisationsformen und Regelungsstrukturen jenseits des Marktes verwiesen, um Probleme von Marktmechanismen wie Transaktionskosten zu analysieren. ${ }^{18}$ Generell wurde aus dieser Perspektive dann die Rolle

9 Gunnar Folke Schuppert, Staat als Prozess. Eine staatstheoretische Skizze in sieben Aufzügen, Baden-Baden 2010, S. 104. Siehe auch Rudolf Dolzer, Good Governance: Neues transnationales Leitbild der Staatlichkeit?, ZaöRV 64 (2004), S. 535-546.

10 Werner Jann, Governance als Reformstrategie - Vom Wandel und der Bedeutung verwaltungspolitischer Leitbilder, in: Gunnar Folke Schuppert (Hg.), Governance-Forschung, Vergewisserung über Stand und Entwicklungslinien, Baden-Baden 2. Auflage 2006, S. 21; vgl. auch Franz Nuscheler, Good Governance. Ein universelles Leitbild von Staatlichkeit und Entwicklung? INEF-Report 96/2009, Institut für Entwicklung und Frieden, Universität Duisburg-Essen, Duisburg 2009.

11 Gunnar Folke Schuppert, Governance - auf der Suche nach Konturen eines, , anerkannt uneindeutigen Begriffs", in: Gunnar Folke Schuppert / Michael Zürn (Hg.), Governance in einer sich wandelnden Welt, PVS-Sonderband, Wiesbaden 2008, S. 13-40.

12 Michael Zürn, Regieren jenseits des Nationalstaates, Frankfurt/M. 1998.

13 Vgl. auch Nuscheler 2009 (Fn. 10).

14 Renate Mayntz, Über Governance. Institutionen und Prozesse politischer Regelung, Frankfurt/M. 2009; Fritz Scharpf, Games Real Actors Play: Actor-Centered Institutionalism in Policy Research, Boulder 1997.

15 Renate Mayntz, Governance im modernen Staat, in: Arthur Benz (Hg.), Governance - Regieren in komplexen Regelsystemen. Eine Einführung, Wiesbaden 2004, S. 72.

16 Ronald Coase, The Nature of the Firm, in: Economica 4 (1937), S. 386-405.

17 Oliver Williamson, The Economic Institutions of Capitalism, New York, 1985.

18 Birger Priddat, Economic Governance, in: Gunnar Folke Schuppert (Hg.), Governance-Forschung. Vergewisserung über Stand und Entwicklungslinien, Baden-Baden 2. Auflage 2006, S. 173-194. 
von institutionellen Rahmenbedingungen für wirtschaftliche Entwicklung betont. ${ }^{19}$ Anknüpfend an diese institutionenökonomischen Überlegungen entwickelte schließlich die Weltbank die vierte Wurzel des Governance-Begriffes. ${ }^{20}$ In einer Studie über afrikanische Entwicklungsprobleme tauchte 1989 zum ersten Mal prominent der Begriff „Governance“ auf, definiert als ,,a public sector that is efficient, a judicial system that is reliable, and an administration that is accountable to its public". ${ }^{21}$ Die Analyse von (afrikanischen) Ursachen für Entwicklungsblockaden und -fehlschlägen führte zu allgemeinen Vorschlägen zur Verbesserung vorrangig staatlicher institutioneller Rahmenbedingungen, die letztlich Entwicklung ermöglichen sollten. Aus diesen Vorschlägen wurde dann „Good Governance” im Sinne eines „sound development management”. ${ }^{22}$ Seit den 1990er Jahren etablierte sich der Begriff „Good Governance" als bis heute umstrittenes Leitbild in der entwicklungstheoretischen und -politischen Diskussion, da nach wie vor stark diskutiert wird, was „Good Governance“ beinhaltet. Jonas Wolff spricht gar vom Vorwurf des „Neo-Kolonialismus bzw. (Kultur-)Imperialismus“, der im Raum stehe. ${ }^{23}$ Albrecht Stockmayer fasst die Positionen elegant zusammen, wenn er von ,einer Welt, die zu wissen meint, was ,good“ ist und einer anderen Welt, die nicht unbedingt die Auffassungen des Nordens über dessen Universalien teilt oder teilen will“ spricht. ${ }^{24}$ Verbindet man die Stränge der Governance-Diskussion mit den jeweils zu Grunde liegenden Governance-Konzepten, so kann man mit Renate Mayntz zwei Begriffe von Governance sehen, einen sehr weiten und eher analytisch neutralen, wie er in der Politikwissenschaft vorherrscht, und einen engeren, ,zumindest latent normativ akzentuierten“, 25 wie er in der Weltbank zu finden ist. Das Bestreben der Weltbank, Entwicklungserfolge von Ent-

19 Vgl. Douglass North, Institutionen, institutioneller Wandel und Wirtschaftsleistung, Tübingen 1992; zur Institutionenökonomik allgemein siehe Stefan Voigt, Institutionenökonomik, München 2. Auflage 2009.

20 Vgl. Thomas Fuster, Die "Good Governance" Diskussion der Jahre 1989 bis 1994. Ein Beitrag zur jüngeren Geschichte der Entwicklungspolitik unter spezieller Berücksichtigung der Weltbank und des DAC, Bern/Stuttgart/Wien 1998; Nuscheler 2009; World Bank, Weltentwicklungsbericht 2002: Institutionen für Märkte schaffen, Washington D.C./Bonn 2002.

21 World Bank, Sub-Saharan Africa. From Crisis to Sustainable Growth. A long-term-perspective study, Washington D.C. 1989, S. xii.

22 World Bank, Governance and Development. Washington D.C. 1992, S. 1; vgl. Herrmann Hill, Good Governance - Konzepte und Kontexte, in: Gunnar Folke Schuppert (Hg.). Governance-Forschung. Vergewisserung über Stand und Entwicklungslinien. Baden-Baden 2. Auflage 2006, S. 220-250; $B$. C. Smith, Good Governance and Development, Houndsmill 2007. Siehe auch Dolzer 2004 (FN 9).

23 Jonas Wolff, Die externe Förderung von Demokratie und Good Governance zwischen Dominanz und Konvergenz, in: Hans-Jürgen Burchardt, (Hg.), Nord-Süd-Beziehungen im Umbruch. Neue Perspektiven auf Staat und Demokratie in der Weltpolitik, Frankfurt/M. 2009, S. 241.

24 Albrecht Stockmayer, Governance - aus der Praxis der GTZ, in: Gunnar Folke Schuppert (Hg.), Governance-Forschung. Vergewisserung über Stand und Entwicklungslinien. Baden-Baden 2. Auflage 2006, S. 252; siehe auch Anita Ernstorfer / Albrecht Stockmayer (Hg.), Capacity Building for Good Governance, Baden-Baden, 2009; Nuscheler 2009; Heribert Weiland, Ingrid Wehr / Matthias Seifert (Hg.), Good Governance in der Sackgasse, Baden-Baden 2009.

25 Mayntz 2009 (Fn. 14), S. 47; vgl. auch Markus Adam, Die Entstehung des Governance-Konzepts bei Weltbank und UN. Die EZ wird politischer, E+Z 10 (2000), S. 272-274. 
wicklungsländern zu bewerten, führte dann zu Messversuchen und schließlich zur Entstehung der WGI.

\section{Rule of Law, Governance und die World Governance Indicators}

„Better data for better Governance“26 - mit diesem Schlagwort startete die Weltbank daher Ende der 1990er Jahre mit den WGI einen ambitionierten Versuch, im weitesten Sinne Entwicklungsfortschritte bzw. Faktoren, die für Entwicklungsfortschritte verantwortlich gemacht wurden, zu messen. Die WGI sollen es ermöglichen, die Qualität der Regierungsführung eines Landes zu evaluieren, und zwar sowohl im Vergleich mit anderen Ländern als auch im Vergleich über die Zeit. ${ }^{27}$ Letztendlich geht es um Anreize und Konditionalitäten, Entwicklungshilfeleistungen sollten an Entwicklungserfolge geknüpft werden, zuallererst an gute Regierungsführung, an Good Governance. Die Autoren der WGI, Kaufmann und andere, definieren Governance recht kurz und vage als, the traditions and institutions by which authority in a country is exercised“. ${ }^{28}$ Drei Faktoren sind damit gemeint, die in jeweils zwei Dimensionen gemessen werden: Der Prozess, durch den Regierungen gewählt, überwacht und ersetzt werden, erfasst durch „Voice and Accountability“ sowie durch „Political Instability and Violence“; die Fähigkeit von Staaten, ihre Politiken durchzusetzen, erfasst durch „Government Effectiveness“ und „Regulatory Burden“; der Respekt, den Bürger und Staaten für die Institutionen bzw. Regeln zeigen, die soziale Interaktion regulieren, erfasst durch „Rule of Law“ und „Graft". 29

Eine genauere Begründung oder Herleitung der Konzeption von Governance findet sich nicht, es wird sehr pragmatisch vorgegangen. Die WGI stehen aber ganz offensichtlich im Kontext der oben erwähnten Weltbankpublikationen. Es ist deutlich zu sehen, welch zentrale Rolle Rule of Law spielt, wie auch von Kaufmann und Kraay als Kernelement von Governance formuliert: ,the importance of a capable state operating under the rule of law". ${ }^{30}$

Aufbauend auf und anknüpfend an diese institutionenökonomische Position der Weltbank entstanden eine Reihe von empirischen Studien über Zusammenhänge von Institutionen bzw. Rule of Law und Entwicklung. Die grundlegende Argumentation ist relativ einfach und prägnant formuliert von Acemoglu, Johnson und Robinson: „Economic institutions matter for economic growth because they shape the incentives of key economic actors in society, in particular, they influence investments in physical and human capital and technology, and the

26 World Bank, A Decade of Measuring the Quality of Governance, Washington D.C. 2006, S. 1.

27 Vgl. World Bank 2006 (Fn. 26), S. 2.

28 Daniel Kaufmann, Aart Kraay / Pablo Zoido-Lobatón, Governance Matters, Policy Research Working Paper 2196, Washington D.C. 1999, S. 1; ebenso Daniel Kaufmann, Aart Kraay / Massimo Mastruzzi, Governance Matters VIII: Aggregate and Individual Governance Indicators 1996-2008, Policy Research Working Paper 4978, Washington D.C. 2009, S. 5.

29 Vgl. Kaufmann et al. 1999 (Fn 28), S. 1 f.

30 Daniel Kaufmann / Aart Kraay, Governance Indicators: Where are we, where should we be going?, in: The World Bank Research Observer 23, 1 (2008), p. 1-30. 
organization of production...differences in economic institutions are the major sources of cross-country differences in economic growth and prosperity.". ${ }^{31}$ Als konkretes Beispiel für ökonomische Institutionen werden immer wieder Eigentumsrechte bzw. die Wahrscheinlichkeit von Enteignung genannt, die hier eine zentrale Rolle spielen und eng mit Rechtsstaatlichkeit verbunden sind. ${ }^{32}$ Je stärker Rechtsstaatlichkeit ausgeprägt ist, umso geringer die Wahrscheinlichkeit von Enteignung und damit umso sicherer Eigentumsrechte. Je sicherer Eigentumsrechte, desto höher die Erwartungssicherheit ökonomischer Akteure und damit die Bereitschaft zu ökonomischer Aktivität. Explizit vermerkt Daniel Kaufmann, lange Jahre Direktor am World Bank Institute, einem think tank der Weltbank, mittlerweile Senior Fellow der Brookings Institution, in einem Artikel mit dem Titel „Rule of Law Matters“: ,....rule of law does causally impact economic development and growth, particularly in the long term“. ${ }^{33}$ Mit dieser Position vertritt Kaufmann keine Einzelmeinung, wie der Forschungsüberblick von Haggard, MacIntyre und Tiede zeigt. ${ }^{34}$ Empirische Studien, etliche davon aus dem Umfeld der Weltbank, untermauern diese Argumente und greifen für ihre Untersuchungen auf die WGI-Daten zurück, so Easterly und Levine, Kaufmann und Kraay, Rodrik, Subramanian und Trebbi sowie Rigoban und Rodrik, die beiden letztgenannten Studien explizit die Dimension Rule of Law der WGI. ${ }^{35}$

Dies zeigt die zunehmende Bedeutung der WGI. Die WGI haben sich aber nicht nur in der entwicklungstheoretischen und insbesondere entwicklungsökonomischen Diskussion, sondern auch in der entwicklungspolitischen Praxis zu einem (umstrittenen) Referenzpunkt entwickelt. Die Presse wie auch Entwicklungsorganisationen und staatliche Stellen nutzen die Daten zu Vergleichszwecken. Die Millenium Challenge Corporation (MCC) beispielsweise zieht explizit die WGI heran, um Entwicklung zu beobachten und damit die Auswahl von Förderländern, also direkt Mittelzuweisungen, zu legitimieren, ohne allerdings die Problematik der WGI in Rechnung zu stellen. Von 17 Kriterien der MCC sind fünf deckungsgleich mit fünf der sechs Governance-Dimensionen. ${ }^{36}$ Rule of Law, in der MCC-Terminologie „Ru-

31 Daron Acemoglu, Simon Johnson / James A. Robinson, Institutions as a fundamental cause of longrun growth, in: Philippe Aghion / Steven Durlauf (eds.), Handbook of Economic Growth, Volume 1A (2005), S. 389.

32 Vgl. Haggard et al. 2007 (Fn. 6).

33 Daniel Kaufmann, Rule of Law Matters, Northwestern University Law Review Colloquy, Symposium: The Future of law and Development, Part IV, 104 (2010), S. 249.

34 Stephan Haggard, Andrew MacIntyre / Lydia Tiede, The Rule of Law and Economic Development, Annual Review of Political Science 11 (2008), S. 205-234.

35 Vgl. William Easterly / Robert Levine: Tropics, Germs, And Crops: How Endowments Influence Economic Development, NBER Working Paper No. 9106 (2002); Daniel Kaufmann / Aart Kraay, Growth without Governance. Policy Research Working Paper 2928, November 2002, Washington D.C 2002; Dani Rodrik, Arvind Subramanian / Francesco Trebbi, Institutions Rule: The Primacy of Institutions over Geography and Integration in Economic Development, in: Journal of Economic Growth 9, 2 (2004), S. 131-165; Roberto Rigobon / Dani Rodrik, Rule of law, democracy, openness, and income. Estimating the interrelationships, in: Economic of Transition 13, 3 (2005), S. 533-564.

Vgl. $M C C$, Millenium Challenge Corporation: Selection Criteria (2011). 
ling Justly“, spielt eine zentrale Rolle, MCC nutzt die anscheinend präzisen Werte der WGI, um ihre Mittelvergabe als transparent und nachvollziehbar zu loben, was als „hallmark“, als Gütezeichen verkauft wird. ${ }^{37}$ Zwar findet sich in den WGI mehrfach der Hinweis, die Daten sollten bzw. werden nicht als Grundlage für die Mittelvergabe der Weltbank genutzt, so etwa in den Anmerkungen der Abbildungen, die aus dem Internet geladen werden können. ${ }^{38} \mathrm{Al}-$ lerdings erscheint das eher wie das Kleingedruckte im Anhang von komplizierten Verträgen, das nicht wirklich gelesen werden soll, sondern nur zu Alibizwecken aufgeführt wird. Es erscheint daher angebracht, die Messung von Rechtsstaatlichkeit durch die WGI einer genaueren Betrachtung zu unterziehen.

\section{Die Messung von Rule of Law}

Die WGI bilden methodisch immer noch den state of the art der Messung von Rule of Law im Kontext von (Good) Governance. ${ }^{39}$ (Good) Governance wird in sechs Dimensionen operationalisiert, anhand von Clustern zahlreicher Einzelindikatoren. Durch deren Aggregation und Gewichtung wird letztlich (Good) Governance erfasst und vermessen.

In der ersten Version von 1999 wurden etwa 300 Einzelindikatoren von 13 Quellen herangezogen, die 173 Ökonomien abbildeten. ${ }^{40}$ Die WGI wurden zwischen 1999 und 2004 zweijährlich, seit 2004 jährlich weiterentwickelt. 2009 erschien die letzte gedruckte Version, seitdem gibt es nur noch jährliche Updates online auf der Homepage der World Governance Indicators. Für das Update 2011 wurden genau 306 Einzelindikatoren aus 30 verschiedenen Quellen herangezogen, die insgesamt 213 Ökonomien erfassen (vgl. Tabelle 1). ${ }^{41}$

37 Vgl. MCC (Fn. 36).

38 Hierfür nutzt die Weltbank nicht die WGI, sondern die Country Policy and Institutional Assessments CPIA (vgl. Christiane Arndt / Charles Oman, The Politics of Governance Ratings, Working Paper MGSoG/2008/WP003, Maastricht University (2008)). Bei den CPIA handelt es sich um Primärbeurteilungen von Ländern anhand ausgewählter Indikatoren, die in den Meta-Index der WGI eingehen, aber auch umgekehrt von den WGI beeinflusst sein könnten, wie Kritiker befürchten, da es sich bei beiden um eine Bewertung von Governance handelt (vgl. Christiane Arndt / Charles Oman, Uses and Abuses of Governance Indicators, Paris 2006).

39 Vgl. Marie Besançon, Good Governance Rankings. The Art of Measurement, WPF Reports 36, Cambridge, MA 2003; Todd Landman, Map-Making and Analysis of the Main International Initiatives on Developing Indicators on Democracy and Good Governance. Eurostat Contract No. 200221200005 , Final Report, Essex 2003.

40 Vgl. Kaufmann et al. 1999 (Fn. 27). Die Autoren verwenden absichtlich den Begriff "Ökonomien", da es sich nicht immer um allgemein anerkannte "Staaten" im völkerrechtlichen Sinne handelt, zum Beispiel Taiwan.

41 Vgl. die Homepage der WGI unter http://info.worldbank.org/governance/wgi/index.asp (letzter Zugriff 17.11.2011). 
Tab. 1: Governance- Messung der Weltbank

\begin{tabular}{|l|l|l|l|l|l|}
\hline Titel & Jahr & $\begin{array}{l}\text { Erhebungs- } \\
\text { jahr }\end{array}$ & $\begin{array}{l}\text { Anzahl der Ein- } \\
\text { zelindikatoren }\end{array}$ & $\begin{array}{l}\text { Anzahl der } \\
\text { Quellen }\end{array}$ & $\begin{array}{l}\text { Anzahl der } \\
\text { Ökonomi- } \\
\text { en }\end{array}$ \\
\hline GM & 1999 & 1996,1998 & ,über 300” & „Vielzahl” & „über 150” \\
\hline GM II & 2002 & 2000 & 194 & 17 & 175 \\
\hline GM III & 2003 & 2002 & 250 & 25 & 199 \\
\hline GM IV & 2005 & 2003,2004 & 352 & 37 & 209 \\
\hline GM V & 2006 & 2005 & 276 & 31 & 213 \\
\hline GM VI & 2007 & 2006 & 310 & 33 & 212 \\
\hline GM VII & 2008 & 2007 & 340 & 35 & 212 \\
\hline GM VIII & 2009 & 2008 & 441 & 35 & 212 \\
\hline Update & 2011 & 2010 & 306 & 30 & 213 \\
\hline
\end{tabular}

Quelle: eigene Erstellung ${ }^{42}$

Bei den Quellen handelt es sich um internationale Umfragen (Surveys) oder um Experteneinschätzungen. Neun Surveys, etwa von Transparency International, Gallup oder dem Latinobarometro, werden benutzt sowie 26 Expertenmeinungen, jeweils neun von NGOs wie Freedom House oder dem Bertelsmann Transformations Index BTI, ebenfalls neun von öffentlichen Stellen wie der Weltbank selbst oder dem US State Department sowie acht von kommerziellen Wirtschaftsinformationsdiensten wie dem Economist Intelligence Unit. ${ }^{43}$ Dabei erfasst natürlich nicht jede Quelle 213 Länder, die Spannbreite reicht von 12 bis 192 Staaten (vgl. Tabelle 2). Zwei Charakteristika sind hervorstechend: Zum einen sind die WGI keine eigenen erhobenen Quellen, sie sind aus sehr vielen Teilindikatoren zusammengesetzt, bilden also einen Meta-Index, zum anderen beziehen sich alle Quellen ausschließlich auf subjektive Einschätzungen, nicht auf „harte“, „objektive“ Daten.

42 Nach Kaufmann et al. 1999 (Fn. 27); Daniel Kaufmann, Aart Kraay / Pablo Zoido-Lobatón, Governance Matters II - Updated Indicators for 2000/2001, Policy Research Working Paper 2772, Washington D.C. 2002; Daniel Kaufmann, Aart Kraay / Massimo Mastruzzi, Governance Matters III: Governance Indicators for 1996, 1998, 2000, and 2002, in: World Bank Economic Review 18 (2004), S. 253-287; Daniel Kaufmann, Aart Kraay / Massimo Mastruzzi, Governance Matters IV: Governance Indicators for 1996-2004. Policy Research Working Paper 3630. Washington D.C. 2005; Daniel Kaufmann, Aart Kraay / Massimo Mastruzzi, Governance Matters V: Aggregate and Individual Governance Indicators 1996-2005, Policy Research Working Paper 4012, Washington D.C. 2006; Daniel Kaufmann, Aart Kraay / Massimo Mastruzzi, Governance Matters VI: Aggregate and Individual Governance Indicators 1996-2006, Policy Research Working Paper 4280, Washington D.C. 2007 c.; Kaufmann et al. 2008 (Fn. 28); Kaufmann et al. 2009 (Fn. 27); Homepage der WGI (Fn. 29).

43 Vgl. Kaufmann et al. 2009 (Fn. 28), S. 29. 
Tabelle 2: Quellen der WGI

\begin{tabular}{|c|c|c|c|c|c|}
\hline Quelle & Kürzel & Typ & $\begin{array}{l}\text { Länder- } \\
\text { reich- } \\
\text { weite }\end{array}$ & $\begin{array}{l}\text { Reprä- } \\
\text { sentativ }\end{array}$ & $\begin{array}{l}\text { RoL-Mes- } \\
\text { sung }\end{array}$ \\
\hline $\begin{array}{l}\text { African Development Bank Country Policy and } \\
\text { Institutional Assessment }\end{array}$ & $\mathrm{ADB}$ & $\begin{array}{l}\text { Expert } \\
(\mathrm{GOV})\end{array}$ & 53 & & $\mathrm{Ja}$ \\
\hline Afrobarometer & AFR & Survey & 19 & & $\mathrm{Ja}$ \\
\hline $\begin{array}{l}\text { Asian Development Bank Country Policy and } \\
\text { Institutional Assessment }\end{array}$ & ASD & $\begin{array}{l}\text { Expert } \\
(\mathrm{GOV})\end{array}$ & 29 & & $\mathrm{Ja}$ \\
\hline Business Enterprise Environment Survey & BPS & Survey & 27 & & $\mathrm{Ja}$ \\
\hline Bertelsmann Transformation Index & BTI & $\begin{array}{l}\text { Expert } \\
(\mathrm{NGO})\end{array}$ & 125 & & $\mathrm{Ja}$ \\
\hline Freedom House Countries at the Crossroads & CCR & $\begin{array}{l}\text { Expert } \\
(\mathrm{NGO})\end{array}$ & 62 & & $\mathrm{Ja}$ \\
\hline $\begin{array}{l}\text { European Bank for Reconstruction and Deve- } \\
\text { lopment Transition Report }\end{array}$ & EBR & $\begin{array}{l}\text { Expert } \\
(\mathrm{GOV})\end{array}$ & 29 & & \\
\hline $\begin{array}{l}\text { Economist Intelligence Unit Riskwire \& Demo- } \\
\text { cracy Index }\end{array}$ & EIU & $\begin{array}{l}\text { Expert } \\
\text { (CBIP) }\end{array}$ & 181 & $\mathrm{Ja}$ & $\mathrm{Ja}$ \\
\hline Freedom House & FRH & $\begin{array}{l}\text { Expert } \\
(\mathrm{NGO})\end{array}$ & 197 & $\mathrm{Ja}$ & $\mathrm{Ja}$ \\
\hline $\begin{array}{l}\text { Transparency International Global Corruption } \\
\text { Barometer Survey }\end{array}$ & GCB & Survey & 80 & & \\
\hline $\begin{array}{l}\text { World Economic Forum Global Competitiven- } \\
\text { ess Report }\end{array}$ & GCS & Survey & 134 & $\mathrm{Ja}$ & $\mathrm{Ja}$ \\
\hline Global Integrity Index & GII & $\begin{array}{l}\text { Expert } \\
(\mathrm{NGO})\end{array}$ & 79 & & $\mathrm{Ja}$ \\
\hline Gallup World Poll & GWP & Survey & 130 & $\mathrm{Ja}$ & $\mathrm{Ja}$ \\
\hline $\begin{array}{l}\text { Heritage Foundation Index of Economic Free- } \\
\text { dom }\end{array}$ & HER & $\begin{array}{l}\text { Expert } \\
(\mathrm{NGO})\end{array}$ & 179 & $\mathrm{Ja}$ & $\mathrm{Ja}$ \\
\hline $\begin{array}{l}\text { Cingranelli Richards Human Rights Database } \\
\text { and Political Terror Scale }\end{array}$ & HUM & $\begin{array}{l}\text { Expert } \\
(\mathrm{GOV})\end{array}$ & 192 & $\mathrm{Ja}$ & $\mathrm{Ja}$ \\
\hline IFAD Rural Sector Performance Assessments & IFD & $\begin{array}{l}\text { Expert } \\
(\text { GOV) }\end{array}$ & 90 & & $\mathrm{Ja}$ \\
\hline IJET Country Security Risk Ratings & IJT & $\begin{array}{l}\text { Expert } \\
\text { (CBIP) }\end{array}$ & 185 & $\mathrm{Ja}$ & \\
\hline Institutional Profiles Database & IPD & $\begin{array}{l}\text { Expert } \\
(\mathrm{GOV})\end{array}$ & 85 & $\mathrm{Ja}$ & $\mathrm{Ja}$ \\
\hline IREEP African Electoral Index & IRP & $\begin{array}{l}\text { Expert } \\
(\mathrm{NGO})\end{array}$ & 53 & & \\
\hline Latinobarometro & LBO & Survey & 18 & & $\mathrm{Ja}$ \\
\hline $\begin{array}{l}\text { International Research and Exchanges Board } \\
\text { Media Sustainability Index }\end{array}$ & MSI & $\begin{array}{l}\text { Expert } \\
(\mathrm{NGO})\end{array}$ & 76 & & \\
\hline International Budget Project Open Budget Index & OBI & $\begin{array}{l}\text { Expert } \\
(\mathrm{NGO})\end{array}$ & 85 & & \\
\hline
\end{tabular}




\begin{tabular}{|c|c|c|c|c|c|}
\hline Quelle & Kürzel & Typ & $\begin{array}{l}\text { Länder- } \\
\text { reich- } \\
\text { weite }\end{array}$ & $\begin{array}{l}\text { Reprä- } \\
\text { sentativ }\end{array}$ & $\begin{array}{l}\text { RoL-Mes- } \\
\text { sung }\end{array}$ \\
\hline $\begin{array}{l}\text { World Bank Country Policy and Institutional } \\
\text { Assessment }\end{array}$ & PIA & $\begin{array}{l}\text { Expert } \\
(\mathrm{GOV})\end{array}$ & 142 & & $\mathrm{Ja}$ \\
\hline $\begin{array}{l}\text { Political Economic Risk Consultancy Corrupti- } \\
\text { on in Asia Survey }\end{array}$ & PRC & Survey & 15 & & \\
\hline $\begin{array}{l}\text { Political Risk Services International Country } \\
\text { Risk Guide }\end{array}$ & PRS & $\begin{array}{l}\text { Expert } \\
\text { (CBIP) }\end{array}$ & 140 & $\mathrm{Ja}$ & $\mathrm{Ja}$ \\
\hline Reporters Without Borders Press Freedom Index & RSF & $\begin{array}{l}\text { Expert } \\
(\mathrm{NGO})\end{array}$ & 170 & $\mathrm{Ja}$ & \\
\hline $\begin{array}{l}\text { US State Department Trafficking in People Re- } \\
\text { port }\end{array}$ & TPR & $\begin{array}{l}\text { Expert } \\
\text { (GOV) }\end{array}$ & 153 & $\mathrm{Ja}$ & $\mathrm{Ja}$ \\
\hline Vanderbilt University Americas Barometer & VAB & Survey & 23 & & $\mathrm{Ja}$ \\
\hline $\begin{array}{l}\text { Institute for Management and Development } \\
\text { World Competitiveness Yearbook }\end{array}$ & WCY & Survey & 55 & & $\mathrm{Ja}$ \\
\hline $\begin{array}{l}\text { Global Insight Business Conditions and Risk In- } \\
\text { dicators }\end{array}$ & WMO & $\begin{array}{l}\text { Expert } \\
\text { (CBIP) }\end{array}$ & 203 & $\mathrm{Ja}$ & $\mathrm{Ja}$ \\
\hline
\end{tabular}

$\mathrm{CBIP}=$ Commercial Business Information Provider (kommerzielle Wirtschaftsinformationsdienste); GOV $=$ Public Sector Data Provider (staatliche bzw. öffentliche Stellen); NGO = Non-Governmental Organisation Data Provider (Nichtregierungsorganisationen), ausführliche Informationen zu jeder Quelle finden sich im Anhang zu Kaufman, Kraay und Mastruzzi ${ }^{4}$

Quelle: eigene Erstellung ${ }^{45}$

Im Folgenden soll die Rechtsstaatlichkeitsmessung der WGI genauer vorgestellt werden. Rechtsstaatlichkeit oder Rule of Law wird als eine der sechs Dimensionen gemessen. Dabei geht es um die Wahrnehmung von Vertrauen in Regeln und die Gesetzestreue, insbesondere um die Qualität von Vertragssicherheit und um Eigentumsrechte, die Polizei und die Gerichte sowie um Verbrechen und Gewalt: ,[...] perceptions of the extent to which agents have confidence in and abide by the rules of society, and in particular the quality of contract enforcement, property rights, the police, and the courts, as well as the likelihood of crime and violence “. ${ }^{46}$ Auffällig ist, dass Elemente, die oft als Teil von Rechtsstaatlichkeit angesehen werden, etwa der Schutz von Menschen- und Bürgerrechten oder vor Korruption, vom WGI nicht als Bestandteil von Rechtsstaatlichkeit, sondern als eigene Dimensionen von Governance angesehen werden. Für die Messung von Rechtsstaatlichkeit werden insgesamt 73 Indikatoren aus 22 Quellen herangezogen. Neun der Quellen werden als repräsentativ, 13 als nichtrepräsentativ eingestuft. Die Einstufung als nichtrepräsentativ erfolgt nach geographischen und Einkommensverteilungskriterien der untersuchten Länder und ist für die spätere Gewichtung von Bedeutung. Quellen, die nur ausgewählte und eingeschränkte Ländergruppen unter-

44 Kaufmann et al. 2009 (Fn. 28).

45 Nach Kaufmann et al. 2009 (Fn. 28), S. 29.

46 Vgl. Kaufmann et al. 2009 (Fn. 28), S. 6. 
suchen, etwa regionale Surveys wie das Latinobarometro oder das Vanderbilt University Americas Barometer, oder etwa regionale Organisationen wie die Afrikanische Entwicklungsbank, die nur Daten für afrikanische Staaten bieten, gelten als nichtrepräsentativ. Von den neun repräsentativen Quellen der Dimension Rule of Law sind sieben Experteneinschätzungen, zwei Surveys. Von den sieben Experteneinschätzungen stammen drei von kommerziellen Wirtschaftsinformationsdiensten, drei von Regierungsstellen, eine ist von einer NGO.

Tabelle 3: Indikatoren von Rechtsstaatlichkeit der WGI

\begin{tabular}{|l|l|}
\hline Quelle & Indikator \\
\hline Repräsentativ & $\begin{array}{l}\text { Gewaltverbrechen } \\
\text { Organisiertes Verbrechen } \\
\text { Faire Gerichtsverfahren } \\
\text { Durchsetzung von Verträgen } \\
\text { Dauer von Gerichtsverfahren } \\
\text { Konfiszierungen/Enteignungen }\end{array}$ \\
\hline GCS & $\begin{array}{l}\text { Normale Verbrechen führen zu höheren Geschäftskosten } \\
\text { Organisiertes Verbrechen führt zu höheren Geschäftskosten } \\
\text { Qualität der Polizei } \\
\text { Unabhängigkeit der Justiz von Regierung, Bürgern, Unternehmen } \\
\text { Rechtlicher Rahmen zur Beurteilung der Legalität von Regierungs- } \\
\text { handeln ist ineffizient } \\
\text { Geistiges Eigentumsrecht schwach ausgeprägt } \\
\text { Schutz von Finanzeinlagen ist schwach ausgeprägt } \\
\text { Steuerhinterziehung }\end{array}$ \\
\hline GWP & $\begin{array}{l}\text { Vertrauen in die Polizei } \\
\text { Vertrauen in die Justiz } \\
\text { Waren Sie Opfer eines Verbrechens? }\end{array}$ \\
\hline HER & Eigentumsrechte \\
\hline HUM & Unabhängigkeit der Justiz \\
\hline
\end{tabular}




\begin{tabular}{|c|c|}
\hline Quelle & Indikator \\
\hline IPD & $\begin{array}{l}\text { Achtung der Gesetze in Verwaltungsakten } \\
\text { Sicherheit von Personen und Sachen } \\
\text { Organisierte Kriminalität } \\
\text { Bedeutung informeller Ökonomie } \\
\text { Bedeutung von Steuerhinterziehung im formellen Sektor } \\
\text { Bedeutung der Zollvergehen } \\
\text { Funktionieren des Justizsystems } \\
\text { Wahrung traditioneller Eigentumsrechte } \\
\text { Wahrung von Eigentumsrechten } \\
\text { Wahrung von Privatverträgen } \\
\text { Achtung der Regierung von Verträgen } \\
\text { Rechtsverfahren in wirtschaftlichen Streitigkeiten } \\
\text { Geistige Eigentumsrechte } \\
\text { Arrangements zur Wahrung geistiger Eigentumsrechte } \\
\text { Eigentumsrechte in der Landwirtschaft }\end{array}$ \\
\hline PRS & $\begin{array}{l}\text { Law and order: Stärke und Unabhängigkeit der Justiz, Beachtung } \\
\text { von Gesetzen }\end{array}$ \\
\hline TPR & Illegaler Menschenhandel \\
\hline WMO & $\begin{array}{l}\text { Unabhängigkeit der Justiz } \\
\text { Kriminalität }\end{array}$ \\
\hline \multicolumn{2}{|l|}{$\begin{array}{l}\text { Nicht repräsen- } \\
\text { tativ }\end{array}$} \\
\hline ADB & Eigentumsrechte \\
\hline AFR & $\begin{array}{l}\text { Wie leicht oder schwierig ist es nach Ihrer Erfahrung Hilfe von der } \\
\text { Polizei zu bekommen? }\end{array}$ \\
\hline ASD & Rechtsstaatlichkeit \\
\hline BPS & $\begin{array}{l}\text { Justizsystem (Fairness, Unabhängigkeit, Schnelligkeit, Ehrlichkeit, } \\
\text { „Affordability“) } \\
\text { Schutz von Eigentumsrechten } \\
\text { Wie problematisch ist organisierte Kriminalität für das Wachstum } \\
\text { Ihres Unternehmens? } \\
\text { Wie problematisch ist die Justiz für das Wachstum Ihres Unterneh- } \\
\text { mens? } \\
\text { Wie problematisch ist Straßenkriminalität für das Wachstum Ihres } \\
\text { Unternehmens? }\end{array}$ \\
\hline BTI & Rechtsstaatlichkeit \\
\hline CCR & Rechtsstaatlichkeit \\
\hline CPIA & Eigentumsrechte \\
\hline FRH & Justiz (Rahmen und Unabhängigkeit) \\
\hline
\end{tabular}




\begin{tabular}{|l|l|}
\hline Quelle & Indikator \\
\hline GII & $\begin{array}{l}\text { Accountability der Exekutive } \\
\text { Accountability der Justiz } \\
\text { Rechtsstaatlichkeit } \\
\text { Durchsetzung von Gesetzen }\end{array}$ \\
\hline IFD & $\begin{array}{l}\text { Zugang zu Land } \\
\text { Zugang zu Wasser für Landwirtschaft }\end{array}$ \\
\hline LBO & $\begin{array}{l}\text { Vertrauen in die Justiz } \\
\text { Vertrauen in die Polizei } \\
\text { Waren Sie Opfer eines Verbrechens? }\end{array}$ \\
\hline PIA & $\begin{array}{l}\text { Vertrauen in die Justiz } \\
\text { Vertrauen in den Obersten Gerichtshof } \\
\text { Vertrauen in die Polizei } \\
\text { Waren Sie Opfer eines Verbrechens? }\end{array}$ \\
\hline WCY & $\begin{array}{l}\text { Eigentumsrechte und regelbasierte Governance } \\
\text { Steuerhinterziehung ist eine verbreitete Praxis in Ihrem Land } \\
\text { Pie Justiz ist nicht fair } \\
\text { Persönliche Sicherheit und Privateigentum werden nicht ausrei- } \\
\text { Phend geschützt } \\
\text { Parallelwirtschaft beeinträchtigt die wirtschaftliche Entwicklung } \\
\text { Ihres Landes } \\
\text { Geistige Eigentumsrechte werden nicht ausreichend durchgesetzt in } \\
\text { Ihrem Land }\end{array}$ \\
\hline
\end{tabular}

Quelle: eigene Erstellung ${ }^{47}$

Die einzelnen Informationen werden nun mit einem komplexen ökonometrischen Verfahren der Zeitreihenanalyse, dem „Unobserved Components Model“, in den Teilindex der Dimension Rechtsstaatlichkeit umgerechnet. ${ }^{48}$

\section{Ergebnisse und Kritik}

Die Ergebnisse der WGI können hier nicht ausführlich dargestellt werden, alle einzelnen Daten und Ergebnisse sind auf der Homepage einsehbar. Die Indikatoren der einzelnen Dimensionen werden nicht in einen einzelnen Gesamtindex der (Good) Governance zusammengeführt, sondern sind in den jeweiligen Einzeldimensionen abrufbar. Dabei bedienen sich WGI einer interessanten Darstellungsweise. Die Ergebnisse werden in Form eines farbigen

47 Nach der Homepage der WGI (Fn. 40.).

$48 \mathrm{Zu}$ den methodischen Details ausführlich Kaufmann et al. 2009 (Fn. 28), S. 98ff.; Arndt / Oman 2006 (Fn. 38), S. 103ff.; Wolfgang Muno, Die Vermessung der Welt - Eine kritische Analyse der World Governance Indicators der Weltbank, in: Zeitschrift für Vergleichende Politikwissenschaft, Sonderheft 2 (2012), Indizes in der vergleichenden Politikwissenschaft, S. 87-114. 
Rankings dargestellt, wobei Prozentzahlen die Zahl der Länder, die schlechter bzw. besser dastehen, abbilden, und in Form eines erweiterten Ampelschemas wird schon farblich klar gemacht, wo die Länder liegen, im grünen Bereich oder im roten, dazwischen Länder im gelben, orange- und rosafarbenen Bereich. Zusätzlich werden die Varianzen angezeigt. Es ist deutlich zu erkennen, dass die Varianzen beträchtlich und etliche Zuordnungen daher fragwürdig sind. Das eine oder andere Land könnte ebenso gut eine andere Farbe bekommen.

Einige herausragende Ergebnisse für die Regierungsführung verschiedener Länder im Zeitraum von 1998 bis 2008 werden von Kaufmann, Kraay und Mastruzzi kurz erwähnt. ${ }^{49}$ In der Dimension „Rule of Law“ sind die Elfenbeinküste, Ecuador, Bolivien und Zimbabwe die Verlierer, dagegen Georgien, Liberia, Ruanda und Estland Gewinner. Doch was bedeutet beispielsweise die Kategorisierung ,signifikante Verschlechterung“ in der Dimension Rule of Law etwa für Bolivien angesichts der Methodik der Indexkonstruktion? Rein logisch betrachtet existieren drei Möglichkeiten. Erstens könnte sich Boliviens Rechtsstaat im Zeitraum in der Tat verschlechtert haben. Zweitens könnte sich Bolivien nicht verändert, die anderen Länder aber verbessert haben. Somit würde sich Bolivien dadurch relativ verschlechtern und im Index einen schlechteren Rang bekommen. Drittens aber könnte sich Bolivien sogar verbessert haben, wenn sich die anderen Länder jedoch noch deutlicher verbessert hätten, würde sich Bolivien auch hier relativ verschlechtern und im Index abrutschen, obwohl es sich tatsächlich gebessert hätte. Diese Gedankenspielerei offenbart einige Probleme der WGI, es gibt allerdings noch mehr, wie zu zeigen ist. Zunächst ist generell zu konstatieren, dass die WGI zweifellos den umfassendsten Versuch darstellen, Governance und Rechtsstaatlichkeit zu messen. ${ }^{50}$ Es handelt sich wohl um den elaboriertesten Index überhaupt, der in politikwissenschaftlichen Kontexten zu finden ist. Drei Punkte stechen positiv hervor: erstens die äußerst umfangreiche Quellenlage, die eine Vielzahl anderer Versuche, Aspekte von Governance zu messen, erfasst und berücksichtigt; zweitens die mit 213 erfassten Ländern überaus große Reichweite; drittens die Transparenz. Sämtliche technisch-mathematischen Details zur Berechnung der Indizes sind dokumentiert, alle Quellen detailliert aufgelistet, sowohl in verschiedenen Printversionen als auch seit 2005 in umfangreichen Datenbanken

49 Vgl. Kaufmann et al. 2009 (Fn. 28), S. 20.

50 Ein neuer Ansatz zur Rechtsstaatsmessung, der konzeptionell umfassender ist, stellt der Rule of LawIndex des World Justice Projects dar. Mehr als 400 Variablen werden hier für neun Dimensionen von Rechtsstaatlichkeit gemessen, basierend auf Umfragen (über 1000 Befragte pro Land) sowie Experteneinschätzungen. Allerdings ist dieser Index noch sehr neu und wurde 2011 erst zum zweiten Mal erhoben. Dies sowie die geringe Reichweite von bis dato nur 66 untersuchten Ländern reduziert die Anwendbarkeit in quantitativen Studien bisher. Allerdings ist zu erwarten (und angekündigt), dass dieser neue Rule of Law-Index, wenn er bekannter und, wie angekündigt, ausgebaut wird, zukünftig mehr Beachtung und Verwendung finden wird. Vgl. hierzu den World Justice Project Rule of Law Index 2011 unter http://worldjusticeproject.org/sites/default/files/wjproli2011_0.pdf (letzter Zugriff 17.11.2011). Zu weiteren Versuchen der Rechtsstaatsmessung siehe auch Svend-Erik Skaaning, Measuring the Rule of Law, Political Research Quarterly 63, 2 (2010), S. 449-469 sowie Jenniver Sehring, Peter Thiery und Wolfgang Muno, Die Messung von Rechtsstaatlichkeit, in: Josef Estermann (Hg.), Interdisziplinäre Rechtsforschung zwischen Rechtswirklichkeit, Rechtsanalyse und Rechtsgestaltung, Beckenried 2009, S. 211-230. 
auf der Homepage der WGI, wo sich auch sämtliche Ergebnisse abrufen lassen. Kritikpunkte werden ebenfalls offen diskutiert, zum Teil sogar auf der Homepage der WGI selbst. Diese positiven Aspekte dürfen aber auch nicht über einige wesentliche Kritikpunkte hinwegtäuschen. $^{51}$

Die mangelnde theoretische Begründung der Gesamtkonzeption der WGI ist zweifellos der hauptsächliche Schwachpunkt des Indexes. Die allgemeine Definition wird nicht aus einem Forschungskontext heraus aufgestellt, obwohl ganz offensichtlich ein solcher existiert. Keine der präsentierten Dimensionen beinhaltet einen Bezug zur theoretischen Literatur. Die Attribute von Rule of Law sind, wie bereits erwähnt, ,,perceptions of the extent to which agents have confidence in and abide by the rules of society, and in particular the quality of contract enforcement, property rights, the police, and the courts, as well as the likelihood of crime and violence“ ${ }^{52}$ Diese Attribute werden von Kaufmann, Kraay und Mastruzzi nicht weiter erklärt. Trotz der umfangreichen Literatur zu Rechtsstaatlichkeit und Rule of Law ${ }^{53}$ wird kaum eine der Vorleistungen zur Kenntnis genommen oder zur theoretischen Herleitung herangezogen.

51 Zur kritischen Methodendiskussion vgl. Muno 2012 (Fn. 48); Arndt / Oman 2006 (Fn. 38); Kazi Iqbal / Anwar Shah, How do worldwide governance indicators measure up? Washington D.C. 2008; Laura Langbein/Stephen Knack, The Worldwide Governance Indicators and Tautology: Causally related separable concepts, indicators of a common cause, or both? Policy Research Working Paper 4669, Washington D.C. 2008; Stephen Knack / Laura Langbein, The Worldwide Governance Indicators: Six, One, or None?, in: Journal of Development Studies 46, 2 (2010), S. 350-370, die Antwort auf Knack und Langbein von Daniel Kaufmann, Aart Kraay/Massimo Mastruzzi, Response to: "The Worldwide Governance Indicators: Six, One, or None" (2010 a); M. A. Thomas, What do the Worldwide Governance Indicators Measure, in: The European Journal of Development Research, 22, 1 (2010), S. 31-54; die Antwort auf Thomas von Daniel Kaufmann, Aart Kraay / Massimo Mastruzzi, Response to: "What Do the Worldwide Governance Indicators Measure?", in: European Journal of Development Research 22, 1 (2010 b), S. 55-58; die Debatte in The Journal of Politics mit Marcus Kurtz / Andrew Schrank, Growth and Governance: Models, Measures, and Mechanisms, in: The Journal of Politics 69, 2 (2007 a), S. 538-554; Daniel Kaufmann, Aart Kraay / Massimo Mastruzzi, Growth and Governance: A Reply, in: The Journal of Politics 69, 2 (2007 a), S. 555-562; Marcus Kurtz / Andrew Schrank, Growth and Governance: A Defense, in: The Journal of Politics 69, 2 (2007 b), S. 563-569; Daniel Kaufmann, Aart Kraay / Massimo Mastruzzi, Growth and Governance: A Rejoinder, in: The Journal of Politics 69, 2 (2007 b), S. 570-572 sowie den jüngsten methodologischen Beitrag von Daniel Kaufmann, Aart Kraay/Massimo Mastruzzi, The Worldwide Governance Indicators: Methodology and Analytical Issues, World Bank Policy Research Working Paper 5430 (2010 c).

52 Daniel Kaufmann, Aart Kraay/Massimo Mastruzzi 2009 (Fn. 27), S. 6.

53 Siehe etwa Herbert L.A. Hart, The Concept of Law, Oxford 1961; Lon Fuller, The Morality of Law, New Haven; Joseph Raz, The Rule of Law and Its Virtue, 1977 (abgedruckt in Joseph Raz, The Authority of Law, Oxford 2009, S. 210-233); Antonin Scalia, The Rule of Law as a Law of Rules, in: The University of Chicago Law Review 56, 4 (1989), S. 1175-1188; oder Tamanaha 2004, um nur einige Beispiele zu nennen. Aus politikwissenschaftlicher Sicht siehe hierzu etwa Michael Becker, Hans-Joachim Lauth /Gert Pickel (Hg.), Rechtsstaat und Demokratie, Wiesbaden 2001; Guillermo O'Donnell, Democracy, Law, and Comparative Politics, in: Studies in Comparative International Development 36, 1 (2001), S. 7-36; oder Hans-Joachim Lauth/Jenniver Sehring, Putting Deficient Rechtsstaat on the Research Agenda: Reflections on diminished Subtypes, in: Comparative Sociology 8, 2 (2009), S. 165-201. 
Bei näherer Betrachtung erscheint das Vorgehen relativ willkürlich. Um die Anzahl und die inhaltliche Stoßrichtung der Dimensionen und ihrer Attribute begründen zu können, wäre ein Nachweis der Konstruktvalidität notwendig, diese ist aber aufgrund der fehlenden expliziten theoretischen Herleitung und mangelnder Hypothesen schwer zu erbringen. ${ }^{54}$ Auch die Zuordnung der Einzelindikatoren zu den Dimensionen erfolgt nicht theoriegeleitet, sondern, wie die Autoren selbst betonen, nach der subjektiven Einschätzung, ob diese Indikatoren den gleichen Aspekt von Governance messen. Dabei werden diverse Einzelindikatoren mehreren dieser Aspekte zugeteilt, teilweise gehen Einzelindikatoren in alle sechs Dimensionen mit ein. Insgesamt wird also das Verhältnis der Attribute zueinander, zum allgemeinen Konzept und zu den Komponenten überhaupt nicht spezifiziert. Statt die Indikatoren aus einem allgemeinen, theoretischen Konzept abzuleiten, hat es eher den Anschein, als würden Kaufmann und die Koautoren umgekehrt aus der zusammenfassenden Beschreibung der Summe der Einzelindikatoren relativ willkürlich und ad-hoc eine allgemeine Definition konstruieren. ${ }^{55}$

Neben der Konzeptionalisierung, die grundsätzlich kritisiert wird, kann eine Vielzahl von Detailproblemen bei der Umsetzung des Indexes konstatiert werden. ${ }^{56}$ Als problematisch gelten hauptsächlich folgende Punkte: mangelnde Transparenz von Einzelquellen, Fehlerkumulation durch die Gestaltung als Meta-Index, das Verfahren der Gewichtung, das dazu führt, dass die kommerziellen Wirtschaftsinformationsdienste bis zu 71 Prozent Anteil an der jeweiligen Dimension bekommen, die Varianz der Quellen über Zeit und von Land zu Land, die letztlich bedeutet, dass, methodisch gesehen, ein Vergleich der Länder ebenso unzulässig ist wie ein Vergleich eines Landes über die Zeit, da die jeweiligen Samples nicht übereinstimmen, und die ausschließliche Subjektivität der Daten, die zu erheblichen Wahrnehmungsverzerrungen führen kann.

\section{Fazit}

Rechtsstaatlichkeit wird in der institutionenökonomischen Perspektive als ein bestimmender Faktor für Entwicklung gesehen. Theoretische Modellannahmen werden durch etliche empirische Studien diesbezüglich gestützt. Dies zeigt sich insbesondere in der von der Weltbank dominierten Diskussion über Good Governance, die auf institutionenökonomischen Annahmen und Ergebnissen basiert. Eine wichtige Rolle spielt hierbei der Versuch, Good Governance und Rechtsstaatlichkeit zu messen, was durch die World Governance Indicators (WGI) geschieht, die für etliche der empirischen Untersuchungen herangezogen werden. Die Fehlermargen, Abweichungen, Mängel und Grenzen der WGI sind bekannt und, besonders bei der leicht zugänglichen und nutzerfreundlich gemachten graphischen Darstellung, auch offensichtlich für jeden, der sich näher damit beschäftigt. Würde es sich lediglich um Probleme einer rein wissenschaftlichen Diskussion handeln, wäre dieser Aspekt vielleicht vernachläs-

54 Vgl. Thomas 2010 (Fn. 49).

55 Vgl. Thomas 2010 (Fn. 49), S. 41.

56 Vgl. ausführlicher zu diesen methodischen Problemen Muno 2012 (Fn. 46). 
sigbar. Aber die Nutzung des Indexes für praktisch-politische Zwecke wie die Vergabe von Entwicklungshilfegeldern betrifft direkt das Leben zahlreicher Menschen und hat daher auch moralische Implikationen. Moralisch problematisch sind die WGI im Sinne des Philosophen Thomas Pogges, ,weil derart mängelbehaftete Indizes in die Irre leiten und Politikern falsche Anreize setzen $[\ldots]^{657}$ und damit ,unser moralisches Urteil verzerren und die Allokation von Ressourcen durch Regierungen, internationale Behörden und Nichtregierungsorganisationen (NGOs) fehlleiten". 58

Insbesondere die einseitige Verkürzung von Rechtsstaatlichkeit auf Eigentumsrechte kann hierbei kritisiert werden. Es handelt sich um eine sehr „dünne“ Version von Rechtsstaatlichkeit. In der empirischen Umsetzung in den WGI werden zwar noch weitere Indikatoren herangezogen, aber die Gewichtung führt zu einer Dominanz der Daten kommerzieller Wirtschaftsdienste, die bis zu 71 Prozent an den jeweiligen Dimensionen ausmachen. Die Weltbankmitarbeiter Iqbal und Shah kritisieren dies als einseitige Überbewertung, als „Western business perspectives“. ${ }^{59}$ Auch ist festzustellen, dass der Entwicklungsbegriff im Kontext dieser Diskussionen regelmäßig auf Wirtschaftswachstum verkürzt wird. Damit ist der theoretische wie empirische Zusammenhang zwar kohärent, da Eigentumsrechte offensichtlich eine große Wirkung auf Wirtschaftswachstum haben, aber eben sehr stark und einseitig verzerrt. Insbesondere soziale Aspekte von Entwicklung werden ausgeblendet. Länder wie Venezuela oder Bolivien, die seit einigen Jahren wieder eine stark dirigistische und etatistische Wirtschaftspolitik verfolgen und Eigentumsrechte durch Enteignungen und Verstaatlichungen beschneiden, haben zwar klar eine positive soziale Bilanz, Armut und Ungleichheit etwa gehen zurück. Sie werden aber, wie auch gezeigt, schlechter beurteilt als Länder, die sich dem marktorientierten Mainstream der Weltbank beugen, ob die Governance, die Regierungsleistung nun tatsächlich „,besser“ ist oder nicht.

Wenn die Kritik von Iqbal \& Shah an einer „Western business perspective“, die den WGI zu Grunde liegen soll, zutrifft, und dafür spricht einiges, dann würden verzerrte Beurteilungen vorliegen, die zu fehlgeleiteter Allokation führen.

Zudem macht es wenig Sinn, Rechtsstaatlichkeit als Kausalfaktor für Entwicklung isoliert zu betrachten. Es verwundert wenig, wenn Rechtsstaatsförderung, neues und zentrales Leitbild entwicklungspolitischer Zusammenarbeit, nur begrenzte Erfolge zeigt. „Jenseits theoretisch-ökonomischer Rechtfertigungen“, so Riegner und Wischmeyer, „fallen ... die messbaren Ergebnisse der neuen Welle an ,rule of law"-Reformen bescheiden aus". ${ }^{60}$ Rechtsstaatlichkeit ist einerseits Bestandteil eines Institutionenkomplexes, der auch Demokratie und Verteilungsfragen umfasst, Rechtsstaatlichkeit basiert andererseits wiederum auf verschiedenen gesellschaftlichen Institutionen, die wiederum auch von historischen Entwicklungen,

57 Thomas Pogge, Die Entwicklung moralisch plausibler Indizes für Armut und Geschlechtergleichstellung: ein Forschungsprogramm, in: Zeitschrift für Politik 56, 3 (2009), S. 325.

58 Pogge 2009 (Fn. 57), S. 327.

59 Iqbal/Shah 2008 (Fn. 51), S. 0.

60 Riegner/ Wischmeyer 2011 (FN 4). 
Akteuren, Akteurskonstellationen und Interessen abhängen. Aus analytischen Gründen mag es nachvollziehbar sein, Rechtsstaatlichkeit als Einzelfaktor für wissenschaftliche Zwecke zu isolieren. Aber einfache Antworten auf komplizierte Entwicklungsfragen sind von solch einer Beschäftigung mit einem reduzierten Rechtsstaatlichkeitsbegriff in der Entwicklungsforschung nicht zu erwarten. 